\title{
Angiopoietin receptor TEK mutations underlie primary congenital glaucoma with variable expressivity
}

\author{
Tomokazu Souma, ${ }^{1,2}$ Stuart W. Tompson, ${ }^{3}$ Benjamin R. Thomson,,${ }^{1,2}$ Owen M. Siggs, ${ }^{4}$ Krishnakumar Kizhatil, ${ }^{5}$ Shinji Yamaguchi, ${ }^{1,2}$ \\ Liang Feng, ${ }^{6}$ Vachiranee Limviphuvadh, ${ }^{7}$ Kristina N. Whisenhunt, ${ }^{3}$ Sebastian Maurer-Stroh, ${ }^{7,8}$ Tammy L. Yanovitch, ${ }^{9}$ \\ Luba Kalaydjieva, ${ }^{10}$ Dimitar N. Azmanov, ${ }^{10,11}$ Simone Finzi, ${ }^{12}$ Lucia Mauri, ${ }^{13}$ Shahrbanou Javadiyan, ${ }^{4}$ Emmanuelle Souzeau, ${ }^{4}$ \\ Tiger Zhou, ${ }^{4}$ Alex W. Hewitt, ${ }^{14,15,16}$ Bethany Kloss, ${ }^{3}$ Kathryn P. Burdon,, ${ }^{4,16}$ David A. Mackey, ${ }^{15,16}$ Keri F. Allen, ${ }^{17}$ Jonathan B. Ruddle, ${ }^{14}$ \\ Sing-Hui Lim, ${ }^{18}$ Steve Rozen, ${ }^{18}$ Khanh-Nhat Tran-Viet, ${ }^{19}$ Xiaorong Liu, ${ }^{6}$ Simon John, ${ }^{5,20}$ Janey L. Wiggs, ${ }^{17}$ Francesca Pasutto, ${ }^{21}$ \\ Jamie E. Craig, ${ }^{4}$ Jing Jin,, ${ }^{1,2}$ Susan E. Quaggin, ${ }^{1,2}$ and Terri L. Young ${ }^{3,18}$ \\ 1Feinberg Cardiovascular Research Institute and 2Division of Nephrology/Hypertension, Northwestern University Feinberg School of Medicine, Chicago, Illinois, USA. \\ ${ }^{3}$ Department of Ophthalmology and Visual Sciences, University of Wisconsin-Madison, Madison, Wisconsin, USA. ${ }^{4}$ Department of Ophthalmology, Flinders University, Adelaide, South Australia, Australia. \\ ${ }^{5}$ The Howard Hughes Medical Institute and the Jackson Laboratory, Bar Harbor, Maine, USA. ${ }^{6}$ Department of Ophthalmology, Northwestern University, Feinberg School of Medicine, Chicago, Illinois, USA. \\ ${ }^{7}$ Bioinformatics Institute, Agency for Science Technology and Research, Singapore. ${ }^{8}$ School of Biological Sciences, Nanyang Technological University, Singapore. ${ }^{9}$ Department of Ophthalmology, \\ Dean McGee Eye Institute, University of Oklahoma, Oklahoma City, Oklahoma, USA. ${ }^{10}$ Harry Perkins Institute of Medical Research and Centre for Medical Research, University of Western Australia, \\ Perth, Western Australia, Australia. "Department of Diagnostic Genomics, PathWest, QEIl Medical Centre, Perth, Western Australia, Australia. ${ }^{12}$ Department of Ophthalmology, \\ Hospital das Clínicas of University of São Paulo, São Paulo, Brazil. ${ }^{3}$ Niguarda Ca'Granda Hospital, Milan, Italy. ${ }^{14}$ Centre for Eye Research Australia, University of Melbourne, \\ Royal Victorian Eye and Ear Hospital, Melbourne, Victoria, Australia. ${ }^{15}$ Centre for Ophthalmology and Visual Science, University of Western Australia, Lions Eye Institute, Perth, Western Australia, Australia. \\ ${ }^{16}$ Menzies Institute for Medical Research, University of Tasmania, Hobart, Tasmania, Australia. ${ }^{17}$ Department of Ophthalmology, Massachusetts Eye and Ear Infirmary, Harvard Medical School, \\ Boston, Massachusetts, USA. ${ }^{18}$ Duke-National University of Singapore Graduate Medical School, Singapore. ${ }^{19}$ University of California Medical Center, Sacramento, California, USA \\ ${ }^{20}$ Department of Ophthalmology and Sackler School of Graduate Biomedical Sciences, Tufts University School of Medicine, Boston, Massachusetts, USA. \\ ${ }^{21}$ Institute of Human Genetics, Friedrich-Alexander-Universität Erlangen-Nürnberg, Erlangen, Germany.
}

\begin{abstract}
Primary congenital glaucoma (PCG) is a devastating eye disease and an important cause of childhood blindness worldwide. In PCG, defects in the anterior chamber aqueous humor outflow structures of the eye result in elevated intraocular pressure (IOP); however, the genes and molecular mechanisms involved in the etiology of these defects have not been fully characterized. Previously, we observed PCG-like phenotypes in transgenic mice that lack functional angiopoietin-TEK signaling. Herein, we identified rare TEK variants in 10 of 189 unrelated PCC families and demonstrated that each mutation results in haploinsufficiency due to protein loss of function. Multiple cellular mechanisms were responsible for the loss of protein function resulting from individual TEK variants, including an absence of normal protein production, protein aggregate formation, enhanced proteasomal degradation, altered subcellular localization, and reduced responsiveness to ligand stimulation. Further, in mice, hemizygosity for Tek led to the formation of severely hypomorphic Schlemm's canal and trabecular meshwork, as well as elevated IOP, demonstrating that anterior chamber vascular development is sensitive to Tek gene dosage and the resulting decrease in angiopoietin-TEK signaling. Collectively, these results identify TEK mutations in patients with PCG that likely underlie disease and are transmitted in an autosomal dominant pattern with variable expressivity.
\end{abstract}

\section{Introduction}

Glaucoma is a group of heterogeneous diseases that is characterized by a chronic degenerative optic neuropathy, affecting more than 60 million people worldwide (1-3). Primary congenital glaucoma (PCG) (OMIM 231300) is a severe form of the disease with unclear etiology and is characterized by infant/early-childhood ocular hypertension, enlarged eye globes (buphthalmos), and optic neuropathy, which can result in vision loss and blindness - often

Authorship note: T. Souma and S.W. Tompson, as well as S.E. Quaggin and T.L. Young, contributed equally to this work.

Conflict of interest: The authors have declared that no conflict of interest exists. Submitted: December 16, 2015; Accepted: April 19, 2016.

Reference information: J Clin Invest. 2016;126(7):2575-2587. doi:10.1172/JCI85830. despite treatment (4-6). Indeed, PCG accounts for $18 \%$ of children enrolled in institutions for the blind worldwide (7). PCG occurs in all ethnic groups, but the disease incidence varies according to ethnic background, ranging from 1:1,250 in inbred populations to 1:30,000 in populations with heterogeneous ethnicity $(6,8-12)$. Families can exhibit autosomal recessive or dominant inheritance, although the majority of cases appear to be sporadic $(5,6,10-14)$.

The molecular etiology of PCG is only partially understood, as only a few genes responsible for PCG have been identified (6, 10-14). Mutations in CYP1B1, which encodes a cytochrome $P 450$ enzyme, is the most common cause of autosomal recessive PCG worldwide, accounting for up to $87 \%$ of familial cases in some inbred populations but only $25 \%-27 \%$ in populations with heterogeneous ethnicity $(5,9,15-18)$. With CYP1B1 mutations account- 
A

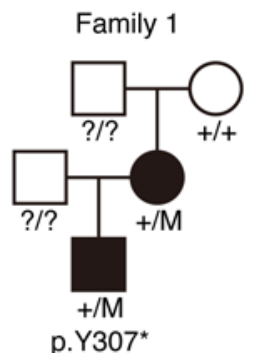

Family 5

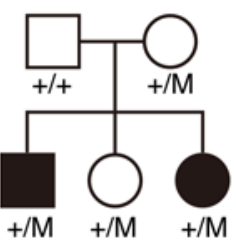

p.Y611C
Family 2

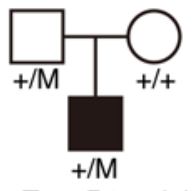

p.T19_R210del
Family 3

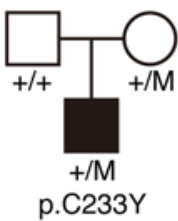

Family 4

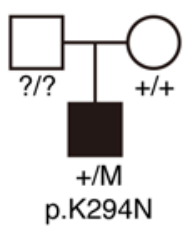

Family 10

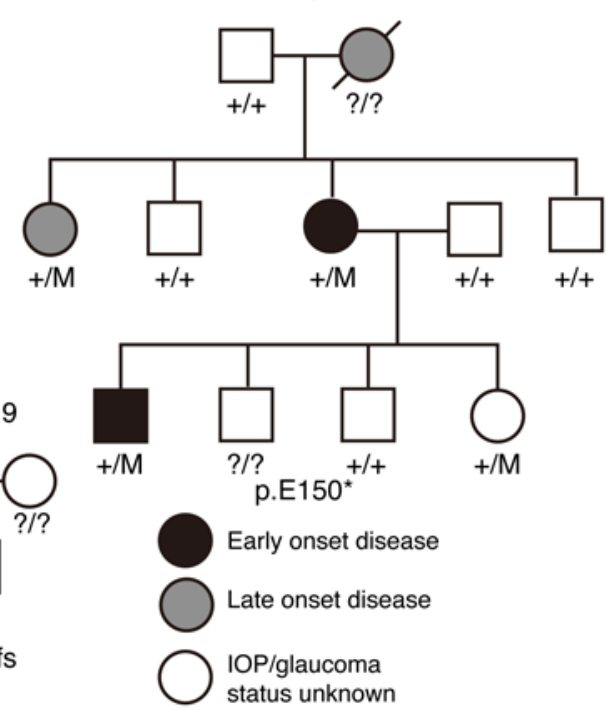

B
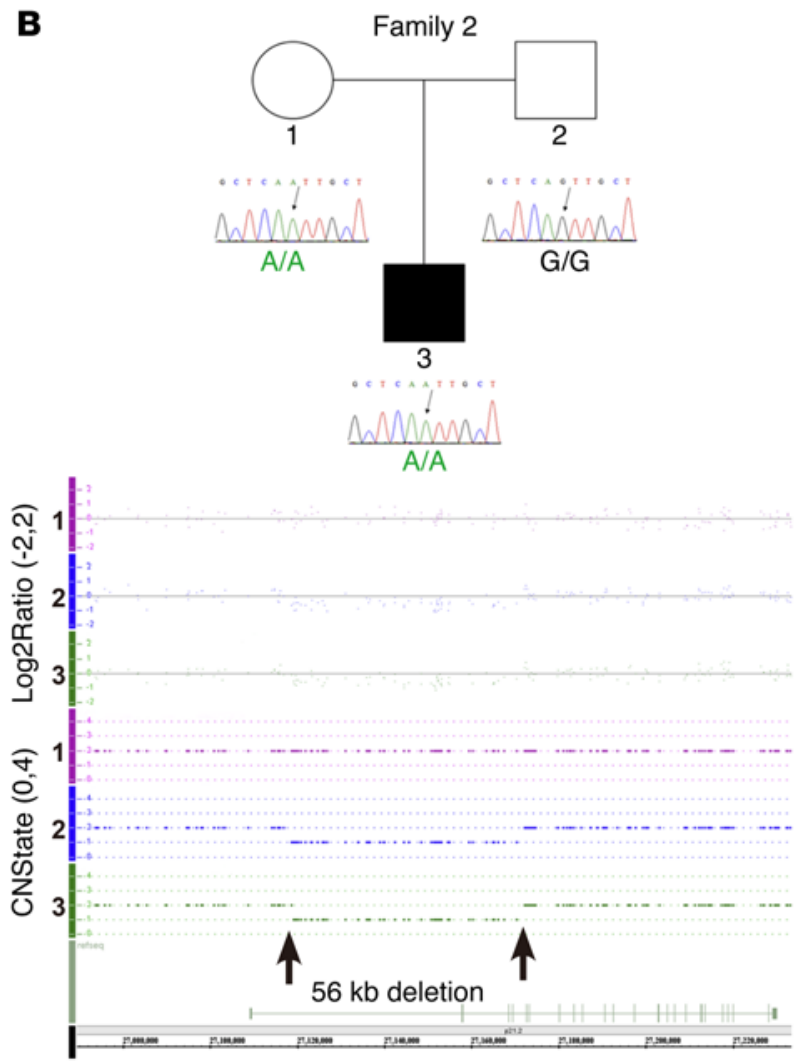

Family 6

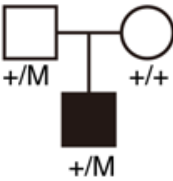

p.G984*
Family 7

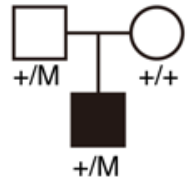

c. $760+2 \mathrm{~T}>\mathrm{C}$

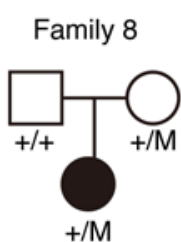

c.3300+2delT

Family 9

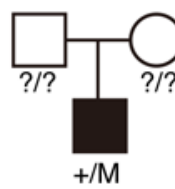

p.K745fs

C

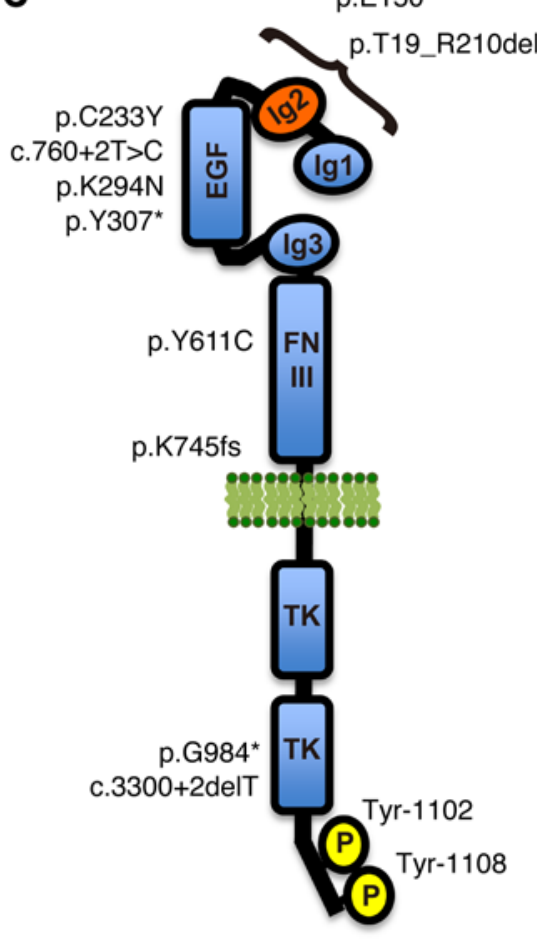

Figure 1. Pedigree structures and TEK mutations identified in $\mathbf{1 0}$ families. (A) Pedigrees of the 10 families with TEK mutations. Specific mutations in TEK are listed below the different pedigrees, with those family members annotated as +/M. Affected individuals are indicated by solid black/gray symbols (black for early-onset disease and gray for late-onset disease; see Table 1 for details). Note: White symbols do not exclude an undiagnosed late-onset disease. Unknown genotypes are marked as ?/?. (B) In family 2, a TEK SNP was inconsistent with father-to-son transmission (upper panel). Copy number variation analysis identified a 56-kb deletion that was passed from the unaffected father (annotated as 2; lower panel) to the unilaterally affected son (annotated as 3). The physical location of the TEK gene is shown, with arrows indicating the deletion breakpoints within introns 1 and 4 . CN, copy number. (C) A schematic illustration of the TEK receptor with the locations of identified variants. Eight of 10 variants were identified in the ectodomain of the receptor. Tyr1102/ Tyr1108 are the primary tyrosine phosphorylation sites in the C-terminal tail of the TEK receptor that initiate downstream signaling events (35, 36).

ing for PCG in less than a third of patients with heterogeneous ethnicity, alternate mechanisms must underlie the majority of these other cases. Furthermore, the mechanism of PCG due to mutations in CYP1B1 is not clearly understood.
It is well recognized that elevated intraocular pressure (IOP) is the key risk factor contributing to glaucoma, including PCG (1-3, $6,19,20)$. The elevated pressure in PCG is believed to result from defects in the aqueous humor outflow (AHO) pathway, rather than 
Table 1. TEK variants identified in 10 PCG families

\begin{tabular}{|c|c|c|c|c|c|c|c|c|c|c|c|c|}
\hline ID & Ethnicity & Sex & $\begin{array}{c}\text { Eyes } \\
\text { affected }\end{array}$ & $\begin{array}{c}\text { Age at } \\
\text { onset }\end{array}$ & $\begin{array}{c}\text { Age of } \\
\text { unaffected } \\
\text { carrier(s) }\end{array}$ & $\begin{array}{l}\text { Chromosome } \\
\text { position }\end{array}$ & Exon(s) & $\begin{array}{l}\text { Coding DNA } \\
\text { mutation }\end{array}$ & $\begin{array}{l}\text { Protein } \\
\text { alteration }\end{array}$ & $\begin{array}{c}\text { ExAC } \\
\text { variant } \\
\text { count }\end{array}$ & $\begin{array}{c}\text { ExAC total } \\
\text { alleles }\end{array}$ & $\begin{array}{c}\text { ExAC ethnically } \\
\text { matched } \\
\text { alleles }\end{array}$ \\
\hline 1 & Lat & $F \& M$ & $U \& U$ & $\begin{array}{c}\text { Infant \& } 4 \\
\text { months }\end{array}$ & - & 27180257 & 7 & c. $9211>A$ & NMD or p.Y307* & Novel & 121,226 & 11,524 \\
\hline 2 & Rom & M & U & $\mathrm{n} / \mathrm{a}$ & $\mathrm{n} / \mathrm{a}$ & $\begin{array}{l}\text { g.(27116823_27118707)_ } \\
\text { (27170308_27172125)del }\end{array}$ & $2-4$ & c.53_628del & p.T19_R210del & - & - & - \\
\hline 3 & Eur (Am) & M & U & Birth & $25 \mathrm{yr}$ & 27172683 & 5 & c.698G $>A$ & p.C233Y & Novel & 121,002 & 66,602 \\
\hline 6 & Eur (I) & M & U & $5 \mathrm{mo}$ & $39 \mathrm{yr}$ & 27213554 & 18 & c. $2950 \mathrm{G}>\mathrm{T}$ & NMD or p.G984* & Novel & 121,294 & 66,698 \\
\hline 7 & Eur (Au) & M & B & Birth & $54 \mathrm{yr}$ & 27172747 & 5 & c. $760+2 T>C$ & $\begin{array}{c}\text { NMD or } \\
\text { p.A254Gfs }{ }^{* 3}\end{array}$ & Novel & 119,718 & 65,966 \\
\hline 8 & Eur $(\mathrm{Au})$ & $\mathrm{F}$ & B & Birth & $38 \mathrm{yr}$ & 27228305 & 22 & c.3300+2delT & p.Y1068Pfs*3 & Novel & 120,580 & 66,356 \\
\hline 9 & Eur $(\mathrm{Au})$ & M & B & Birth & $\mathrm{n} / \mathrm{a}$ & 27204931 & 14 & c.2232dupG & $\begin{array}{c}\text { NMD or } \\
\text { p.K745Efs }{ }^{*} 76\end{array}$ & Novel & 121,392 & 66,726 \\
\hline
\end{tabular}

Ethnicity: Lat, Latino; Rom, Romani; Eur, European; Afr, African; I, Italian; Am, American; Au, Australian. Sex: M, Male; F, Female. Eyes affected: U, unilateral; B, bilateral. $\mathrm{n} / \mathrm{a}$, data not available. NMD, nonsense-mediated decay of mRNA transcript predicted (no protein product). Chromosome position in accordance with GRCh37/hg19 assembly. TEK mRNA reference sequence NM_000459.4. TEK protein reference sequence NP_000450.2.

an increase in aqueous humor $(\mathrm{AH})$ production $(5,6,14)$. Ocular anterior chamber fluid ( $\mathrm{AH})$ is produced by the ciliary body and drained mainly through Schlemm's canal (SC) and uveoscleral pathways $(21,22)$. An imbalance in fluid homeostasis results in elevated IOP. In humans, $80 \%$ of fluid drainage is conducted through the conventional, or trabecular, AHO pathway (23). AH first passes through the trabecular meshwork (TM) before entering the lymphatic-like SC for drainage $(22,24,25)$. The remaining $20 \%$ of fluid egresses via the unconventional uveoscleral tract pathway, which includes the extracellular spaces within the iris, ciliary muscle, and sclera (21).

The angiopoietin receptor TEK (tunica interna endothelial cell kinase, also known as Tie2) is a receptor tyrosine kinase and regulates vascular homeostasis through its auto- and transphosphorylation (26-29). The primary ligand, angiopoietin-1 (ANGPT1), is expressed by pericytes and other vascular supporting cells, and exerts its proangiogenic and vascular stabilizing effects through activating TEK. A second ligand, ANGPT2, is secreted by the endothelium and acts as a context-dependent partial agonist of $\operatorname{TEK}(26,27,29,30)$.

In the eye, the TEK receptor is highly expressed in the SC endothelium $(24,25)$. Recently, we discovered that angiopoietin-TEK signaling is required for SC development in mice (31). Deletion of TEK or both major angiopoietin ligands after embryonic day 16.5 circumvents embryonic lethality and results in developmental loss of SC, extremely elevated IOP, rapid and complete retinal ganglion loss, and glaucoma (31). Despite the critical role of this signaling pathway for SC development in mice, the relevance of this pathway in human disease has not, to our knowledge, been previously reported.

Here, we describe human mutations in TEK identified in a PCG cohort of 189 families that do not carry mutations in the known disease-causing genes CYP1B1 (16-18), LTBP2 (32), FOXC1 (33), and MYOC (13). We identified 10 heterozygous novel/rare
TEK variants as disease-causing mutations and assessed their effects on TEK function. We also determined in mice whether haploinsufficiency for Tek is sufficient to result in abnormal SC development and elevation of IOP. In addition to the identification of a pathway for human glaucoma, we assign key functional roles to specific domains of the TEK receptor.

\section{Results}

TEK mutations are identified in PCG families with unknown etiology. To identify novel PCG causative genes, we applied whole exome sequencing to a multiethnic cohort of PCG families with unknown molecular etiology. We performed an initial exome sequencing analysis on 21 affected individuals from 20 families including 2 affected individuals in family 1 (Figure 1A) and 19 sporadic cases. Under a monogenic recessive inheritance model, filtering for novel nonsynonymous, homozygous, or compound heterozygous variants in a single gene identified no candidate genes. An analysis of 2 complete family trios also excluded the possibility of de novo mutations being causative for the phenotype in these 2 families. We initially focused our analysis on a family demonstrating direct parent-to-child transmission of the disease (family 1, Figure 1A), looking for novel shared heterozygous variants, and identified heterozygosity for 3 nonsense and 15 missense variants in common. One of the nonsense variants, p.Y307*, was present in TEK (Figure 1A and Table 1).

We reasoned that loss-of-function (LoF) mutations in TEK could be involved in the etiology of PCG, given our findings of a PCG-like phenotype in Angpt1/Angpt2 and Tek cKO mice (31). Therefore, we sought additional novel/rare TEK variants in a collaborative multi-ethnic cohort totaling 209 individuals in 189 families. In family 2 (Figure 1A), we screened for TEK variants by Sanger sequencing and identified an inconsistency in father-toson transmission for a known SNP (see Sanger sequencing traces in Figure 1B) that suggested the presence of a deletion. Copy number variation analysis was performed on the family trio using Affy- 
Table 2. Results of the TEK splicing donor variants analysis

$\begin{array}{lcccc}\begin{array}{l}\text { Splicing donor } \\ \text { variant }\end{array} & \begin{array}{c}\text { Prediction } \\ \text { algorithm }\end{array} & \text { WT } & \text { Mutant } & \text { Variation (\%) } \\ \text { c.760+2T>C } & \text { HSF } & 94.19 & 67.35 & -28.5 \text {, WT site broken } \\ & \text { MaxEnt } & 10.45 & 2.7 & -74.16 \\ \text { c.3300+2delT } & \text { HSF } & 99.05 & 49.22 & -50.31, \text { WT site broken } \\ & \text { MaxEnt } & 11 & -2.48 & -122.55\end{array}$

Functional prediction for splicing donor variants were performed using Human Splicing Finder version 3.0 (HSF3.0). A score of more than 65 for position weight matrices (HSF) and more than 3 for maximum entropy (MaxEnt) is predictive for a functional splice site. Moreover, if the variation score (between WT and mutant) is under $-10 \%$ for HSF and $-30 \%$ for MaxEnt, the tool considers that the mutation affects the splice site. See the predicted effects of the disruption of splice donor sites in Supplemental Figure 3.

metrix SNP 6.0 arrays, which identified a 56-kb deletion encompassing TEK exons 2-4 (p.T19_R210del) that was passed from the father (unknown affection status) to the unilaterally affected son (Figure 1B). In total in our cohort, whole exome and direct Sanger sequencing identified 10 novel/rare heterozygous TEK variants (Figure 1, A-C, and Table 1). Nine of these 10 mutations (p.Y307*, p.T19_R210del, p.C233Y, p.Y611C, p.E150*, p.K745fs, p.G984*, c. $760+2 \mathrm{~T}>\mathrm{C}$, and c.3300+2delT) were absent from publicly available exome databases (Exome Sequencing Project [NHLBI-ESP, http://evs.gs.washington.edu/EVS/], 6,500 exome sequences; Exome Aggregation Consortium [ExAC, http://exac.broadinstitute.org], 60,706 exome sequences) and 203 in-house control exomes (Table 1). The highest allele frequency observed for the rare variant (p.K294N) was 0.0003 in Latino populations (ExAC) (data not shown). In all 7 of these families for which we had whole exome data, the possibility of TEK gene copy number variants (CNVs) accounting for a second mutant allele was assessed using exome read depth coverage comparisons. Read depth data for each of the 23 TEK exons was extracted from the 7 PCG exomes and compared with the average read depth obtained from a number of unrelated same-batch exome-sequenced samples. In all cases, no evidence for additional exon-spanning duplications or deletions could be found (Supplemental Figure 1; supplemental material available online with this article; doi:10.1172/JCI85830DS1).
Four mutations (p.E150*, p.Y307*, p.K745fs, and p.G984*) are predicted to be null alleles likely to result in mRNA degradation via nonsense-mediated decay or truncated proteins (see predicted truncated proteins in Supplemental Figure 2A; EnzymeX [http:// nucleobytes.com/enzymex/] was used for predictions). Furthermore, the in silico predictive tool Human Splicing Finder (HSF 3.0, http://www.umd.be/HSF3/HSF.html) indicated that the two invariant splice donor mutations $(\mathrm{c} .760+2 \mathrm{~T}>\mathrm{C}$ and c.3300+2delT) affect splicing and result in functionally null proteins (Table 2 and Supplemental Figure 3). The strong enrichment for LoF mutations in our PCG cohort (6 of 189) compared with those observed in the ExAC database ( 2 of 60,706 individuals; $P=2.3 \times 10^{-14}$; odds ratio $=995.2$ [95\% confidence interval $=199.5-4,963.0]$; see Methods for details), supports a role for TEK mutations in PCG pathogenesis. Additionally, according to analysis of evolutionary conservation and in silico predictive tools (Polymorphism Phenotyping v2 [PolyPhen-2, http://genetics.bwh.harvard.edu/pph2/]; Sorting Intolerant from Tolerant [SIFT, http://sift.bii.a-star.edu.sg/]; and FoldX [http://foldx.crg.es/, http://foldxyasara.switchlab.org/]), 2 of the 3 missense mutations are also expected to affect protein function (Table 3 and Supplemental Figures 4-6).

TEK mutations identified in PCG patients exhibit LoF. We experimentally tested the functional impact of PCG-associated TEK variants in cell-based assays to determine their effect on TEK protein production, phosphorylation, and trafficking within the cell. As mutations were identified sequentially, we performed analysis of the first 5 mutations discovered in our primary cohort. In our system, WT TEK protein underwent normal baseline autophosphorylation when expressed in HEK293 cells (Figure 2A). As predicted, the p.Y307* mutant $\mathrm{cDNA}$ (identified in family 1) did not produce a full-length intact protein with C-terminal FLAG tag, as the introduced stop codon is upstream of the tag (Figure 2A and Supplemental Figure 7). To test the possibility that the predicted p.Y307 mutant protein is produced and secreted into the medium, we generated a TEK306-Fc-expressing vector. TEK306-Fc protein, which contains the Ig1, Ig2, EGF1, and EGF2 domains, was detected in the medium (Supplemental Figure 2B and Supplemental Figure 7).

The p.T19_R210del ( $\Delta \mathrm{T} \_\mathrm{R}$, family 2 ) and p.C233Y (family 3 ) mutations resulted in distinctly reduced protein levels accompanied by negligible autophosphorylation, making them functional null alleles (Figure 2A). Interestingly, the p.K294N (fam-

\section{Table 3. Functional prediction results for missense TEK variants}

\section{Prediction method}

\section{No. of representative sequences at positions}

233, 294, \& 611

$\begin{array}{lc}\text { SIFT: TEK orthologs } & 64,64,63 \\ \text { SIFT: TEK family } & 141,139,140 \\ \text { SIFT: Blink } & \text { B } \\ \text { PolyPhen-2 } & 102,106,172 \\ \text { FoldX average ddG, Kcal/mol' (SD) } & \text { n/a }\end{array}$

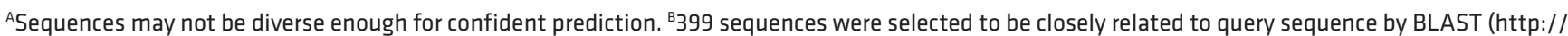
blast.ncbi.nlm.nih.gov/Blast.cgi). 'Average ddG (difference in free energies between mutant and WT protein) run over 5 times. ${ }^{\mathrm{D}} \mathrm{Average}$ ddG from Protein Data Bank (http://www.rcsb.org/pdb/home/home.do) ID: 2GY5. EddG = 2.02 from the structure model that was created using PDB ID: 4N68 as a template.
Prediction result for TEK p.K294N

Affect protein function, $0.0^{A}$

Affect protein function, 0.0

Affect protein function, 0.0

Probably damaging, 1.0

$7.52(3.48)^{0}$

\section{Affect protein function, $0.02^{\mathrm{A}}$}

Tolerated, 0.11

Tolerated, 0.44

Benign, 0.298

$0.93(0.004)^{D}$

\section{Prediction result \\ for TEK p.Y611C}

Affect protein function, $0.0^{A}$

Affect protein function, 0.0

Affect protein function, 0.0

Probably damaging, 1.0 $2.02(0.02)^{\mathrm{E}}$ 
A

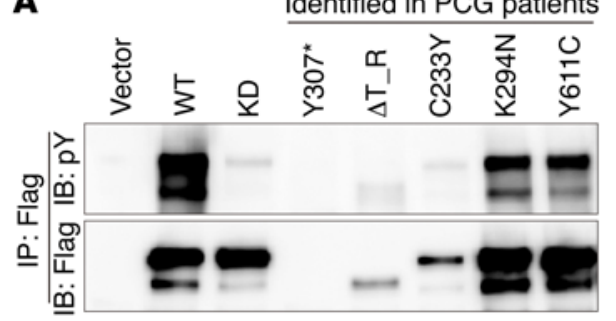

B
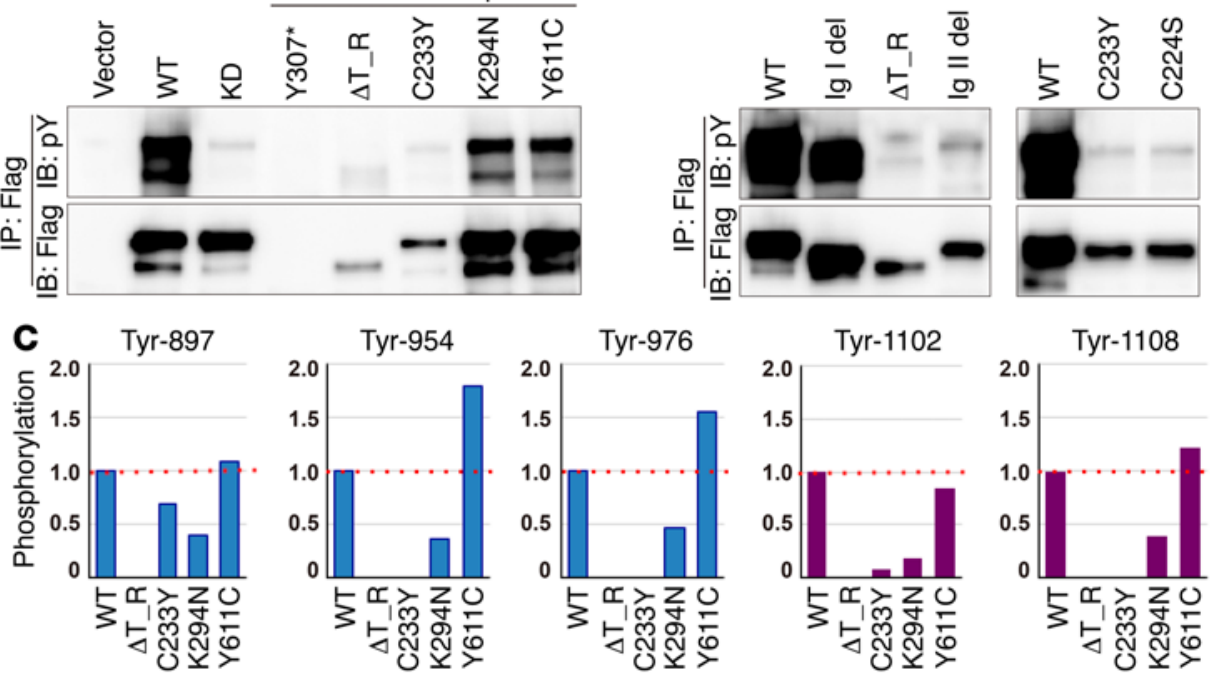

Phosphorylated

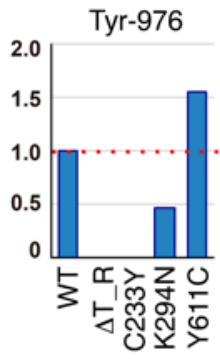

E

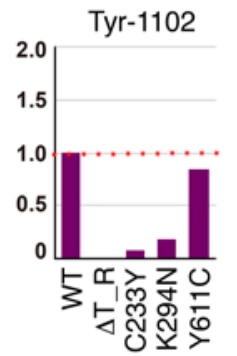

Tyr-1108

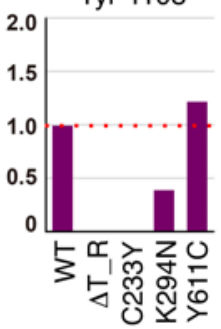

Nonphosphorylated
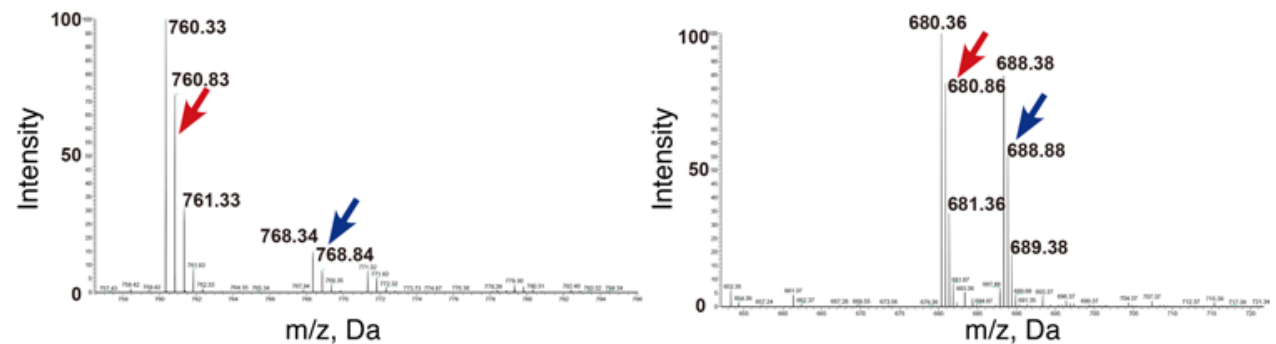

Figure 2. TEK variants lead to reduced autophosphorylation. (A) Western blotting of WT and variant TEK expressed in HEK293 cells, following immunoprecipitation with Flag antibody. (B) The effect of deleting immunoglobulin-like domain (left), and the effect of breaking the disulfide bond formed between C233 and C224 in the EGF I domain (right). (C) Quantitative phosphorylation analysis of each tyrosine residue in the kinase domain (Tyr897,

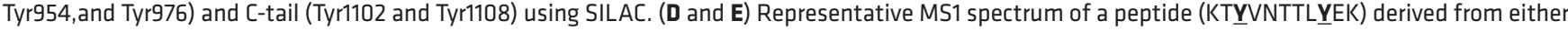
WT (red, light-labeled) or p.K294N mutant (blue, heavy-labeled). Tyrosine residues in this peptide correspond to Tyr1102 and Tyr1108. The spectrum of phosphorylated peptides (KTpYVNTTLPYEK) is shown in $\mathbf{D}$. The spectrum of nonphosphorylated peptides (KTYVNTTLYEK) is shown in E. Igl del, natural variant lacking Ig1 domain; $\triangle \mathrm{T}$ _R, PCG variant lacking Ig1 and Ig2 domain (p.T19_R210del); Igll del, disease-associated variant lacking lg2 domain (34); KD, an artificial kinase-dead mutant (D982A substitution, disrupting DFG motif of kinase domain). Note that both the Igl del variant and the Igll del mutant were not identified in PCG cohorts. All blotting data are representative of more than 3 biological replicates.

ily 4) and p.Y611C (family 5) mutations did not result in reduced protein expression or basal global autophosphorylation in this system (Figure 2A).

Deletion of exons 2-4 (p.T19_R210del, $\Delta \mathrm{T}_{-} \mathrm{R}$ ) results in loss of the terminal 4 aa of the signal peptide sequence (MDSLASLVLCGVSLLLSGTVEG) and the entire Ig1 and Ig2 domains. To determine whether the loss of 4 aa from the signal peptide and/or loss of the Ig1 domain alone is sufficient to cause receptor degradation, we tested a natural splice variant (ENST00000519097) that lacks exon $2-$ encoding the terminal 5 aa of the signal peptide as well as the first Ig domain (Ig1). This splice variant (IgI del) was expressed and phosphorylated at normal levels (Figure 2B). Conversely, a form of TEK mutant carrying an in-frame somatic deletion of exon 3 and part of exon 4 (aa 122-165 of the Ig2 domain [IgII del]) (34) was poorly expressed and phosphorylated, indicating that the Ig2 domain is important for TEK stability and function (Figure 2B, left panels).

As Cys233 forms a disulfide bridge with Cys224 in the EGF1 domain (Supplemental Figures 4 and 5), we hypothesized that the p.C233Y variant may cause misfolding of the protein, due to the disruption of the disulfide bond, followed by degradation. To investigate this possibility, we generated a p.C224S mutant and found that mutation of this paired cysteine led to a similar reduction in protein abundance (Figure $2 \mathrm{~B}$, right panels), confirming the critical importance of the disulfide bond for proper folding and subsequent expression.

Receptor tyrosine kinases mediate their specific signals through distinct tyrosine residues (28), and for TEK-mediated signaling the cytoplasmic C-terminal Tyr1102 and Tyr1108 residues play a central role $(27,35,36)$. To investigate phosphorylation at individual tyrosine residues, we used mass spectrometry together with stable isotope labeling in cell culture (SILAC; Figure 2C and Supplemental Figure 8). While global phosphorylation of the p.K294N mutant was normal, SILAC revealed a 10-fold reduction in Tyr1102 phosphorylation (Figure 2, C-E). This site is known to mediate signaling through p85-PI3K $(35,36)$, suggesting that the p.K294N mutation results in reduced p85-PI3K signaling activity. Based on the critical importance of this site for TEK function (35), we propose that p.K294N is a LoF allele. In contrast, no effect on basal phosphorylation (i.e., ligand-independent phosphorylation) was observed in the p.Y611C mutant (Figure 2C). 
A TEK-FLAG, ZO-1, DAPI
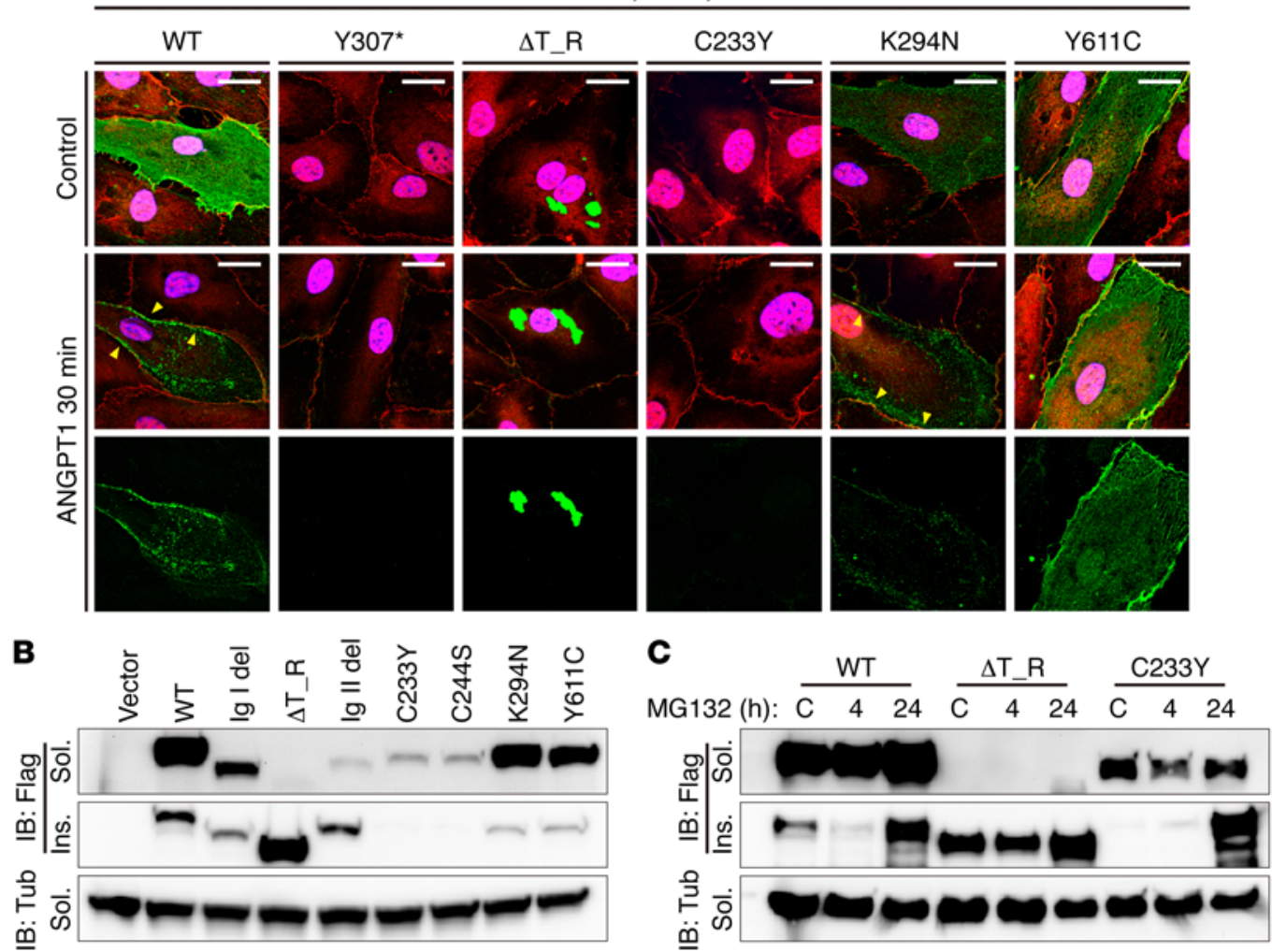

Figure 3. TEK variants lead to protein aggregate formation, reduced expression, and aberrant trafficking. (A) Expression pattern of WT and variant TEK in HUVECs. Transfected HUVECs were stained with anti-Flag (green) and anti-ZO-1 antibody (red). Nuclei were stained with DAPI (pink in merged images). Yellow arrowheads indicate the TEK receptor on cellular junctions. The $Y 307^{*}$ mutant-transfected cells, in which Flag-tag is not expressed, were used as a mock negative control for imaging. Scale bars: $25 \mu \mathrm{m}$. (B) Solubility of TEK variants in HEK293 cells. Solubility of WT and each variant TEK protein was tested by fractionated samples. (C) Effect of proteasomal inhibition on TEK variant expression in HEK293 cells. Cells were treated with MG132, a proteasomal inhibitor, for the indicated times. C, control (DMSO-treated for 24 hours); Sol., soluble fraction; Ins., insoluble fraction; Tub, $\alpha$-tubulin. All data are representative of more than 3 biological replicates.

As portions of the ectodomain are responsible for ligand binding $(37,38)$, subsequent multimerization (38), and subcellular localization (39-41), we predicted that the mutations in these regions could weaken or abolish ligand-mediated activation and signal transduction through the intracellular catalytic domain. Anticipating that TEK ectodomain mutations may affect ligandmediated signaling, we expressed TEK variants in HUVECs and examined their subcellular localization after ligand stimulation. WT TEK was diffusely expressed on the membrane of resting cells (Figure 3A, control WT). When treated with ANGPT1, a major TEK agonist, WT TEK localized to cell-cell junctions and subsequently became internalized (Figure 3A and Supplemental Figure 9), as previously reported $(39,40)$. The p.K294N mutant also followed this dynamic receptor translocation. However, other mutants were unable to respond to ANGPT1 stimulus. p.T19_R210del formed intracellular aggregates, and p.C233Y was not found at the cell membrane (Figure 3A).

WT protein is typically detected within the soluble fraction of a cell lysate (Figure 3B), whereas mutant proteins can form aggregates inside cells and are found within the insoluble fraction. Consistent with subcellular localization studies, p.T19_R210del was found in the insoluble fraction (Figure $3 \mathrm{~B}, \Delta \mathrm{T}_{-} \mathrm{R}$ ). Our results clearly demonstrate that the deletion of Ig1 and Ig2 domains in
$\Delta \mathrm{T} \_\mathrm{R}$ reduces protein solubility, leading to protein aggregate formation, making this allele functionally null. The IgII deletion mutant was found in both fractions, and the p.C233Y mutant, although not insoluble, was less abundant, suggesting that solubility was not the cause of poor expression (Figure 3B). Other variants were largely found in the soluble fraction (Figure 3B).

To determine whether proteasomal degradation underlies the reduced levels of protein, we treated cells expressing the p.T19_R210del or p.C233Y mutant with the proteasomal inhibitor MG132. The inhibitor slightly increased WT protein expression in the soluble fraction. More prominently for WT and p.C233Y, there was a marked increase in insoluble protein following MG132 treatment (Figure 3C). These data suggest that TEK abundance is tightly regulated by proteasome-mediated degradation and that structural defects, such as p.C233Y, lead to enhanced protein degradation through this mechanism.

Interestingly, while the p.Y611C mutant is properly localized to the plasma membrane in resting cells, upon ANGPT1 stimulation it did not follow the normal course of dynamic clustering, junctional localization, and internalization, suggesting that this variant is unable to respond to ligand (Figure 3A, Y611C). In order to measure changes in ligand-mediated TEK phosphorylation, we cotransfected cells with protein tyrosine phosphatase recep- 
A $c .760+2 T>C$

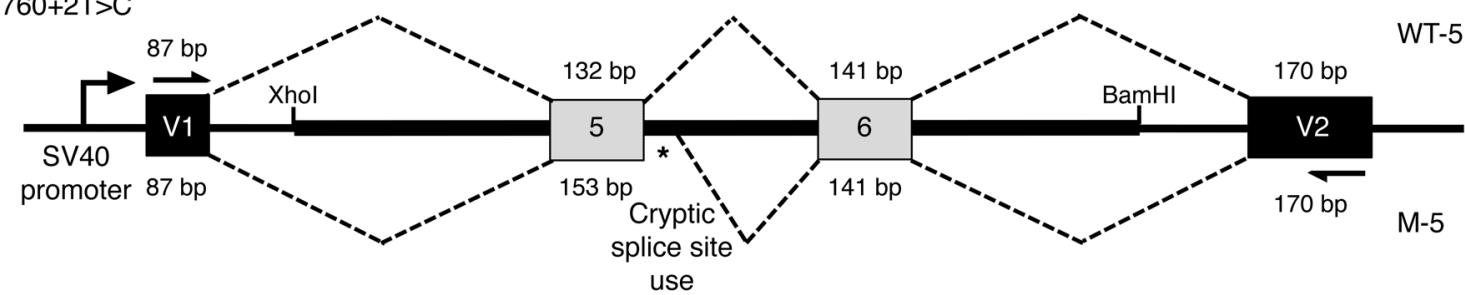

B c.3300+2delT

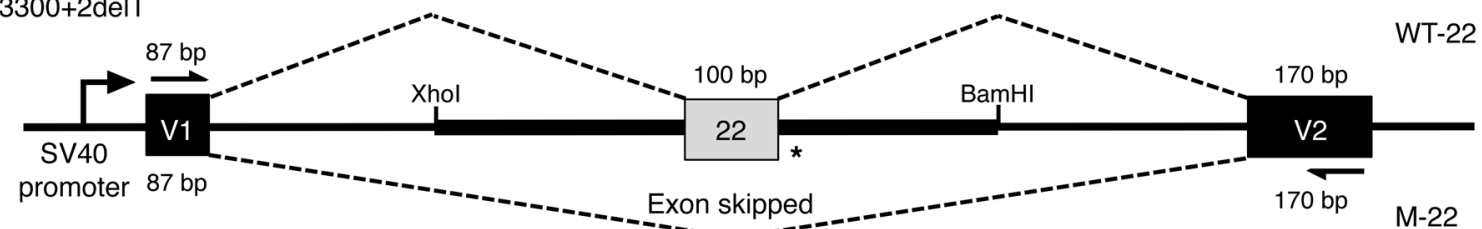

C

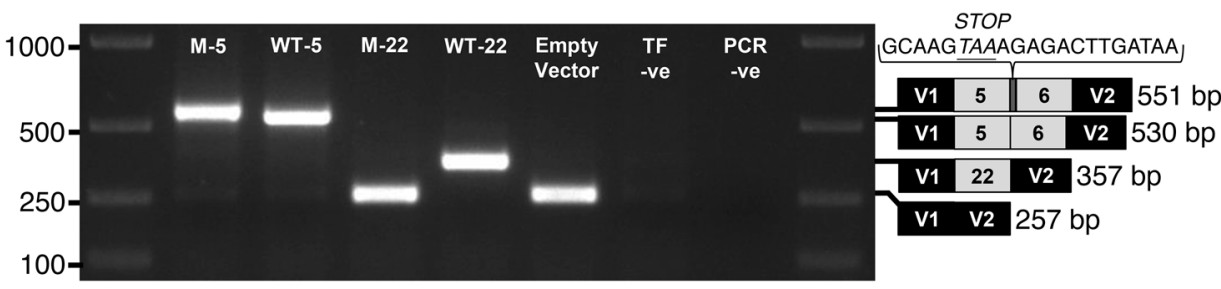

Figure 4. Splice donor site mutations lead to cryptic splice site use or exon skipping. (A and B) Splicing construct minigenes were generated by incorporating genomic regions of the TEK gene into the PSPL3 vector via Xhol and BamHI restriction sites. Vector exons, V1 and V2, are depicted as black boxes and TEK exons 5, 6, and 22 are in gray. WT and mutant splicing products, with included exon sizes in base pairs, are indicated by dashed lines above and below the construct, respectively. The locations of the splice site mutations are shown $\left({ }^{*}\right)$. (A) WT (WT-5) and mutant (M-5) genomic fragments containing TEK exons 5 and 6 were used to model the c.760+2T>C mutation from family 7. (B) WT (WT-22) and mutant (M-22) genomic fragments containing TEK exon 22 were used to model the c.760+2T>C mutation from family 8. (C) Gel electrophoresis of RT-PCR products from transfected Cos-7 cells. Vector exon-specific primers are indicated by arrows in $\mathbf{A}$ and $\mathbf{B}$. TF -ve, cells transfected with PBS only; PCR -ve, PCR-negative control. WT and mutant transcript content, determined by Sanger sequencing, is depicted to the right of the gel image. The additional 21 bp of intron 5 sequence identified within the M5 transcript is shown incorporating a premature termination codon between exons 5 and 6 .

tor type B (PTPRB) to reduce baseline phosphorylation of coexpressed TEK (42). ANGPT1 stimulated the phosphorylation of WT TEK but not p.Y611C, further suggesting a defect in ligand-mediated activation (Supplemental Figure 10).

We also experimentally tested the functional impact of the two splice donor site mutations, c.760+2T>C (family 7) and c.3300+2delT (family 8), in cell-based exon trapping splicing assays to determine their effect on mRNA splicing (Figure 4). In this system, the $\mathrm{c} .760+2 \mathrm{~T}>\mathrm{C}$ mutation eliminated the normal 5 ' splice donor site of exon 5 , which led to a cryptic splice site 21 bp into intron 5 (Figure 4, A and C). As the additional 21 bp sequence incorporated an in-frame termination codon into exon 5 , it was expected to result in mRNA transcript destruction by the nonsense-mediated decay pathway or a severely truncated protein product (p.A254Gfs*3). In contrast, the c.3300+2delT splice donor site mutation resulted in complete skipping of exon 22, effectively removing $100 \mathrm{bp}$ from the mRNA transcript and changing the codon reading frame by which the last exon would be translated (Figure 4, B and C). As a result, exon 23 would encode 2 different amino acid residues (Pro-Thr), followed by a termination codon instead of the normal $57 \mathrm{C}$-terminal residues (p.Y1068Pfs ${ }^{*} 3$ ). Consequently, the mRNA would likely escape destruction by the nonsense-mediated decay pathway, and a protein lacking residues 1068-1124, which includes the C-terminal tyrosine phosphorylation sites (Tyr1102 and Tyr1108), would be produced. As these two tyrosine residues are critical for TEK receptor signaling function $(35,36)$, we propose that the c.3300+2delT mutation represents a LoF allele.

Collectively, our results have demonstrated that 7 of 10 TEK mutations identified in our PCG cohort lead to loss of functional protein by multiple cellular mechanisms - absence of intact protein production (p.Y307*, p.T19_R210del, c.760+2T $>C$ [p.A254Gfs*3], and c.3300+2delT [p.Y1068Pfs*3]), enhanced proteasomal degradation (p.C233Y), impaired phosphorylation of key tyrosine residue (p.K249N), altered subcellular localization and reduced ligand responsiveness (p.Y611C), and protein aggregate formation (p.T19_R210del). We reason that the p.G984* (family 6), p.K745fs (family 9), and p.E150* (family 10) mutations are also likely LoF alleles, as their mRNA transcripts will either be destroyed by the nonsense-mediated decay pathway or encode proteins lacking at least a functional intracellular domain (See Supplemental Figure 2).

TEK haploinsufficiency results in abnormal AHO structure and elevated IOP. After proving that the heterozygous TEK mutations resulted in nonfunctional proteins, we hypothesized that TEK haploinsufficiency is sufficient to cause impaired SC development and ocular hypertension in mice. Using mice harboring a conditional by inversion allele ( $T e k^{\mathrm{COIN}}$ mice) (43), we analyzed AHO drainage pathways in Tek hemizygous and conditional knockout mice. Confocal microscopy revealed that Tek-haploinsufficient mice developed a severely hypomorphic canal with convolutions and focal narrowing, while SC was normal in control mice (Figures 5-7). SC 
A
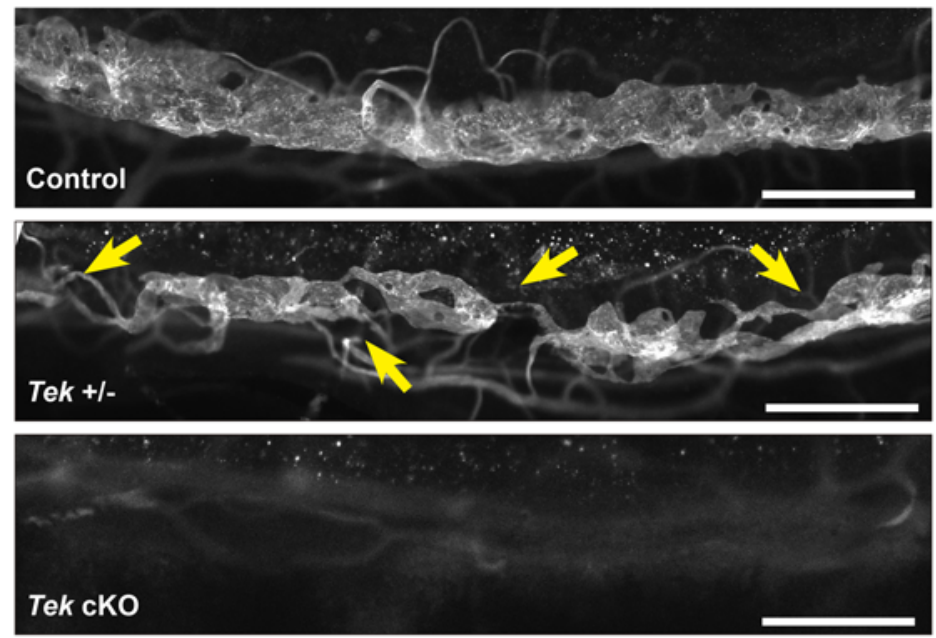

B

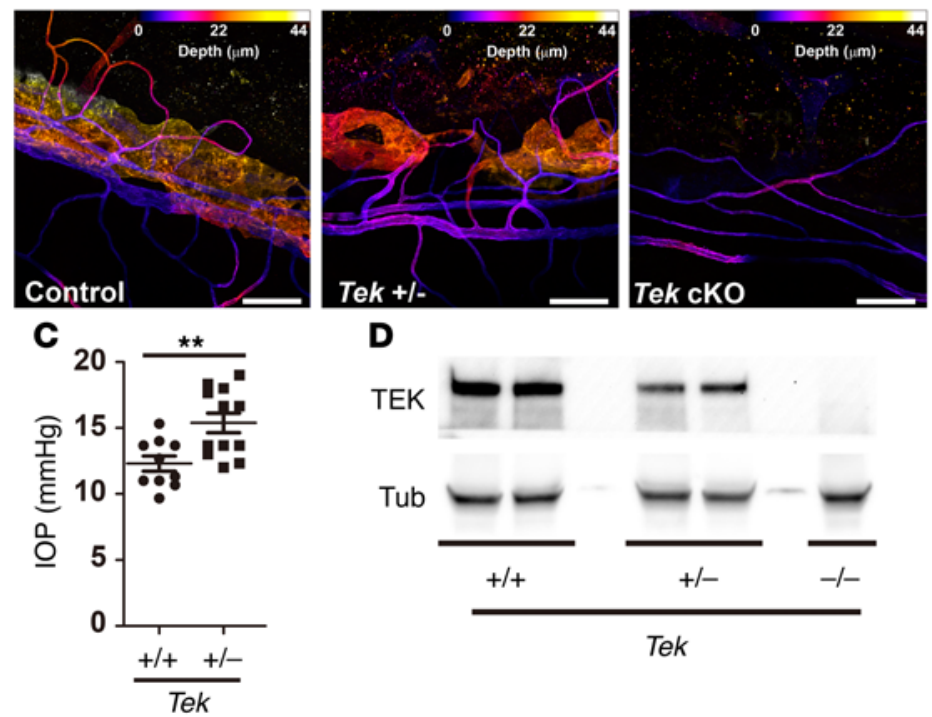

Figure 5. TEK-haploinsufficient mice exhibit hypomorphic SC with elevated IOP. (A and B) Confocal microscopic images of CD31 staining in WT (control), TEK-haploinsufficient ( $\mathrm{Tek}^{+/-}$), and TEK conditional knockout (Tek CKO) mice (10 weeks old). Yellow arrows indicate the convolutions and narrowing of SC. Z-stack $(44 \mu \mathrm{m})$ and pseudo-colored depth projections are shown in B. Scale bars: $25 \mu \mathrm{m}$ (A), $100 \mu \mathrm{m}$ (B) (C) The effect of Tek genotype on IOP. Averaged IOP of both eyes from each mouse is shown. $n=8$ and 9 for $T e k^{+/+}$and $T e k^{+/}$, respectively (25 weeks old). ${ }^{* *} P<0.01$ (2-tailed Student's $t$ test). Error bars indicate SEM. (D) TEK protein expression level of each genotype of mice using lung homogenate.

was completely absent in Tek-knockout mice, clearly demonstrating the absolute requirement and gene dosage sensitivity for TEK during canal development (Figure 5, A and B). Interestingly, quantitative analysis identified the variable degree of hypomorphism in Tek-hemizygous mice (Figure 6). We further analyzed the iridocorneal region using serial histologic sections. Whereas the control WT eyes showed well-developed SC and TM, eyes of Tek-hemizygous mice showed hypoplastic SC and TM, indicating that reduced TEK signaling is detrimental for formation of AHO structures (Figure 7).

To analyze the functional consequence of dysmorphic SC development and hypomorphic TM, we analyzed IOP levels by rebound tonometry. Measurement of IOP confirmed that haploinsufficient mice exhibited a $25 \%$ elevation in IOP (control 12.3
$\mathrm{mmHg}, \mathrm{Te}^{+/-} 15.4 \mathrm{mmHg}, P=0.0046$; Figure 5C and Supplemental Figure 11). The IOP levels were modestly correlated with the degree of SC hypomorphism $(r=0.3703$, $P<0.01)$. These results demonstrate that reduced TEK signaling causes developmental defects of the AHO structure, a key feature of PCG, and correlates with elevated IOP.

\section{Discussion}

Ocular hypertension has been identified as the most important risk factor in human glaucoma, and current glaucoma therapy is focused on lowering IOP using pharmacological or surgical approaches $(1-3,20,44,45)$. While IOP is influenced by both production and drainage of the $\mathrm{AH}$, defects in anterior chamber outflow have been identified as the source of ocular hypertension in glaucoma patients, including PCG patients $(5,46)$. Despite evidence that vascular growth factors are essential for SC development and $\mathrm{AH}$ outflow $(25,31,47)$, links between these pathways and human glaucoma have not been identified. Herein, we describe 10 heterozygous novel/rare protein-altering mutations in TEK as a cause for PCG. Furthermore, our findings provide evidence that TEK gene dosage is critical for the proper development of AHO pathways, and $~ 50 \%$ reduced TEK signaling is sufficient to lead to defective SC development and compromised $\mathrm{AH}$ drainage in mice as well as in patients (Figure 8).

While PCG has clear genetic links, identification of causative alleles is complicated by variable disease penetrance and expressivity $(5,9,12)$. Families identified in our patient cohort were no exception. TEK variants were heterozygous in all affected individuals, and the heterozygous disease allele was vertically transmitted from the affected parent to the affected child in two families (families 1 and 10), clearly indicating dominant transmission of the disease. Interestingly, the development of glaucoma in later decades in two mutation carriers in family 10 (one inferred) and the frequent unilateral involvement observed in mutation carriers (6 of 12 in our cohort with a TEK mutation versus 34 of 209 in our whole cohort) suggest that TEK-related disease can exhibit variable severity and age of onset (Figure 1 and Table 1). This highly variable expressivity may explain the high frequency of carriers without a typical early-onset PCG phenotype in family members. It was not possible to carefully examine many of the parents of the affected probands with PCG for evidence of glaucoma or raised IOP, and given the geographic and historical nature of some of the samples, it is not currently possible to gather this information. With the limited availability of human data, it is difficult to clearly distinguish variable expressivity from reduced penetrance. However, the biological plausibility is underscored by the mouse data clearly showing that haploinsufficiency for Tek is sufficient to lead to functional defects of the AHO pathway with variable expressivity. Therefore, we propose an autosomal dominant model with variable expressivity, consistent with other ocular disorders of developmental origin, such as those caused by mutations in FOXC1, MYOC, PAX6, and OPA1 (6, 10, 48-52). Variable expressivity may be explained by stochastic developmental events 
A
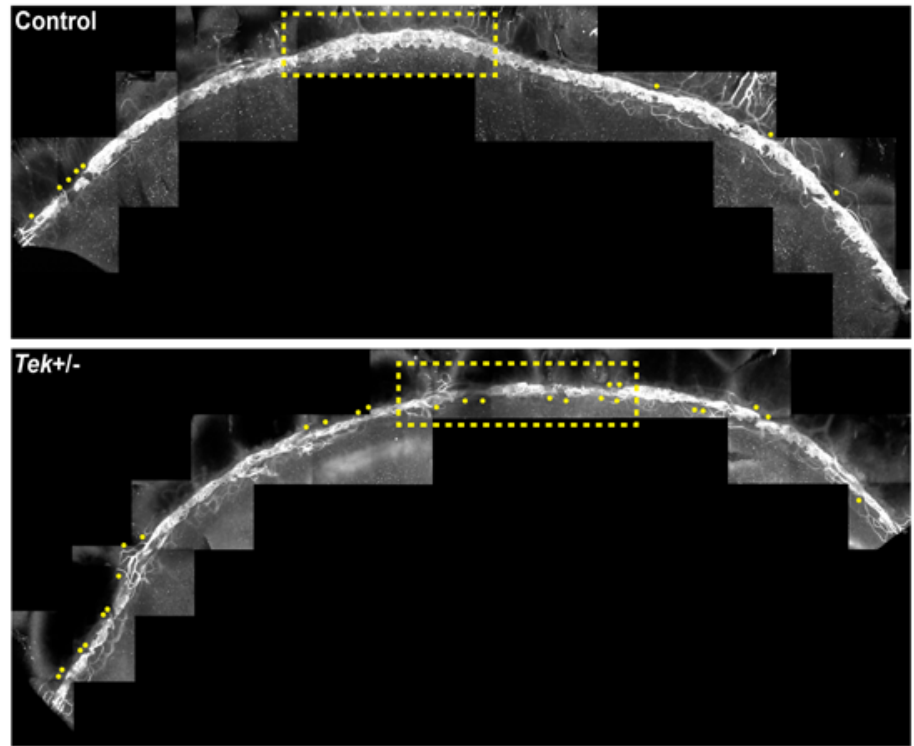

B

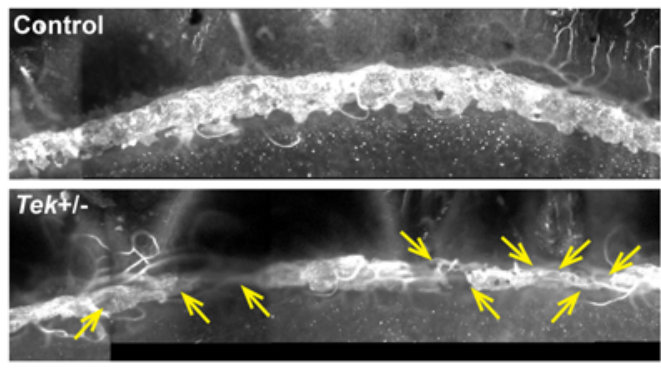

C

SC morphology

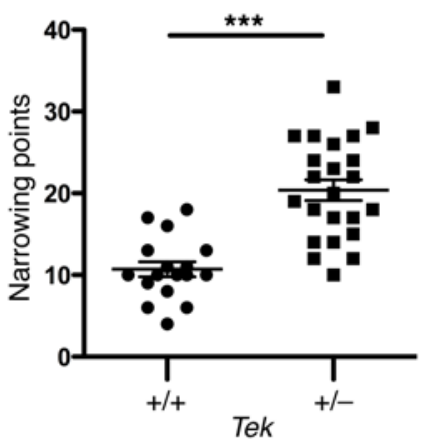

Figure 6. Quantitative analysis of SC morphology. (A) Confocal microscopic images of CD31 staining in control ( $\left.\mathrm{Tek}^{+/+}\right)$and Tek-hemizygous $\left(\mathrm{Tek}^{+/-}\right)$mice. Yellow dots indicate the points with SC narrowing. Original magnification, $x 20$. (B) Higher magnification $(x 60)$ of the yellow dotted area in A. The arrows indicate the narrowing points. (C) Quantitative analysis of SC imaging. The number

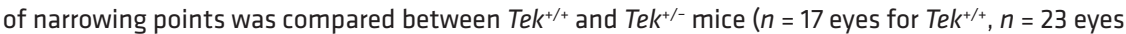
for Tek ${ }^{+/} ; 10-25$ weeks old, littermate controls were used). ${ }^{* *} P<0.001$ (2-tailed Student's $t$ test). Error bars indicate SEM.

in SC formation (25) or oligo-/digenic inheritance. Although we have screened 19 "candidate genes," including the signaling components of the ANGPT-TEK pathway (TEK, ANGPT1, ANGPT2, TIE1, ANGPT4, and PTPRB), genes expressed during SC development (PROX1, FLT4/VEGFR3, and VEGFC), and genes linked to various developmental glaucomas ( $F O X C 2, C Y P 1 B 1, M Y O C$, FOXC1, OPTN, ASB10, TBK1, WDR36, OPA1, and NTF4), we did not identify possible pathogenic variants as a second allele for digenic inheritance. Our results also suggest that ascertaining future PCG subjects for genetic studies should incorporate thorough, longitudinal ophthalmic examinations of reportedly normal parents of affected probands, as they may have subclinical indicators of PCG/glaucoma.

The analysis of PCG mutations in TEK advances our understanding of structure-function relationships within the receptor. Interestingly, all the deletion and missense mutations identified in our PCG cohort were located in the ectodomain and are LoF. The location of PCG mutations is strikingly different from that of the gain-of-function (GoF) TEK mutations that have been linked with hereditary and sporadic venous malformations (VMs) (34, 53, 54). These VM mutations are located solely in the intracellular domain and result in enhanced kinase activity. Furthermore, our cell-based assays have identified new functional roles for various domains. The Ig2 domain, which functions as a ligand-binding domain $(37,38)$, is also critical for stable protein expression, as loss of this Ig domain in p.T19_R210del and IgII del leads to reduced protein levels. The mutation in the EGF1 domain highlights the critical role of cysteine residues, which are needed to disulfide cross-link the protein for stability $(38,55)$. Notably, the mutation in the membrane-proximal fibronectin type III (FN3) domain, which appears in $~ 40 \%$ of all receptor tyrosine kinases (ref. 28 and the Human Protein Reference Database [HPRD], http://www.hprd.org), sheds light on its novel role in ligand-mediated TEK receptor activation. The FN3 domain in the Eph family tyrosine kinase receptors directly stimulates intermolecular interactions to cluster the receptor into higher-order multimers at the plasma membrane (56). These Eph clusters act as efficient signaling centers to trigger cell responses and propagate phosphotyrosine signals. Although the TEK FN3 crystal structure is currently unavailable, the lack of membrane response following ANGPT1 stimulation as observed in HUVECs (Figure 3A) is likely due to impaired signaling activities.

GoF mutations in patients with VMs exhibit variable expressivity and led to the hypothesis that tight regulation of TEK activity is needed for vascular development $(34,54)$. Our findings that hemizygosity for Tek is sufficient to cause disease in mice and that heterozygosity for LoF mutations is associated with PCG of varying severity in patients suggest, for the first time to our knowledge, that there exists a dosage sensitivity for reduced activity of the receptor. It is reported that the conventional outflow path for AH drainage (i.e., SC) plays a less important role in mice than in humans, underscoring the significance of finding elevated IOP in the hemizygous mouse model and suggesting similar structural defects in SC would cause more severe disease in patients (5759). Finally, our data indicate that TEK "dose" might be particularly important for proper development of the vasculature of the anterior chamber of the eye, suggesting that regulation of TEK signal strength may be a therapeutic option.

In summary, we have identified TEK mutations in patients with PCG that follow a dominant inheritance pattern with variable expressivity. Our experimental results further demonstrate that defects in the AHO pathway caused by reduced TEK signaling constitute a new mechanism of PCG disease in humans. It is likely 


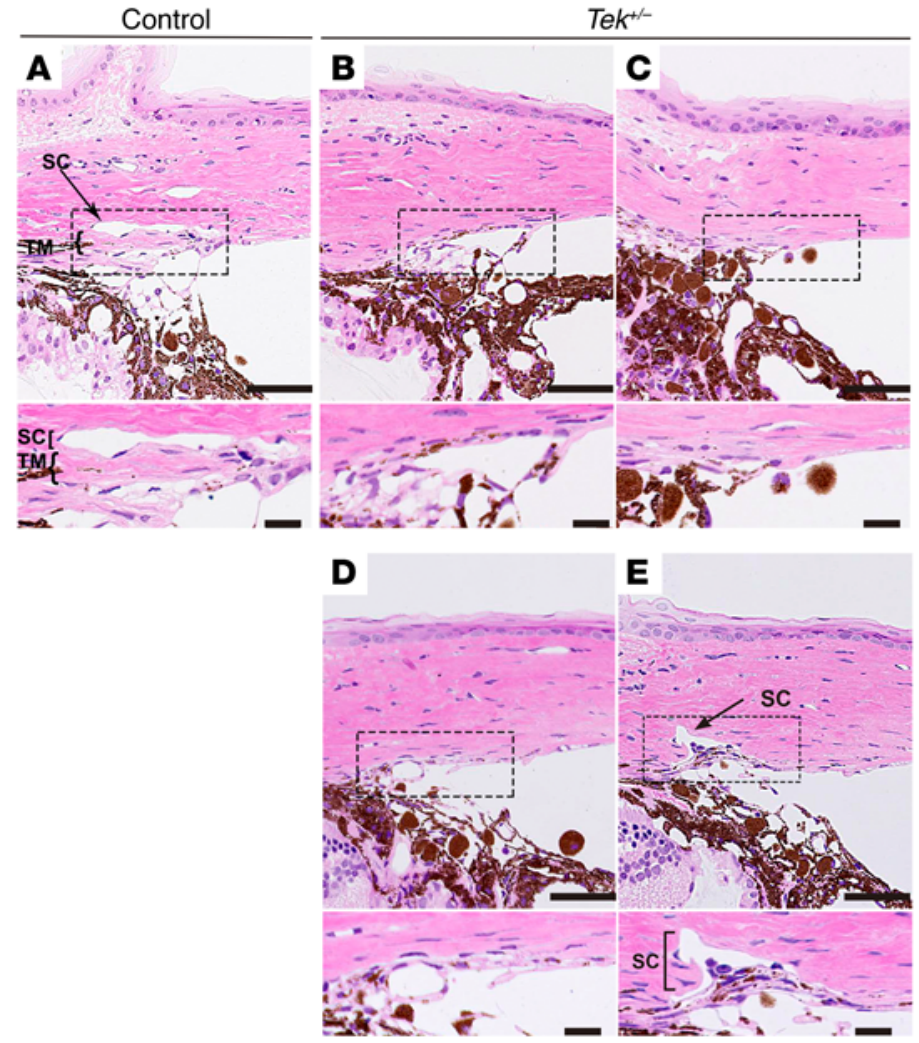

Figure 7. Formation of the SC and TM is defective in Tek-hemizygous mice. (A-E) Histological analysis of iridocorneal angle region from a control eye and $\mathrm{Tek}^{+/-}$mouse eyes (25 weeks old). Upper panels show the location of the SC and TM within the iridocorneal angle. Lower panels show magnified images of the dotted boxes in the upper panels. Note that the TM is hypoplastic and the SC is either absent (B-D) or severely reduced in size (E) in $\mathrm{Tek}^{+/-}$mice. Scale bars: $50 \mu \mathrm{m}$ (upper panels), $10 \mu \mathrm{m}$ (lower panels). that investigating additional genes involved in the formation and maintenance of the AHO pathways will provide further insights to our understanding of this devastating eye disease and aid in the development of new therapeutic strategies for glaucoma.

\section{Methods}

Human study participants. PCG was defined by the following characteristics: (a) age at onset less than 3 years, (b) increased corneal diameter greater than $10 \mathrm{~mm}$ accompanied by corneal edema and/or Haab striae, and (c) IOP greater than $21 \mathrm{mmHg}$ and/or optic nerve cupping greater than 0.4. Any patient with other ocular abnormalities or systemic conditions, other than iris stromal hypoplasia, was excluded from the study. Our studies adhered to the tenets of the Declaration of Helsinki and were compliant with the Health Insurance Portability and Accountability Act.

Whole exome sequencing. Exome sequencing of the initial PCG cohort of 20 families with at least one affected proband was per- formed using a Nimblegen EZ v3 capture kit (Roche) and $2 \times 100$ bp paired-end sequencing on a HiSeq2000 platform (Illumina). All exome variants were validated by Sanger sequencing. The TEK gene was Sanger sequencing screened in an additional 55 families in the primary cohort. An additional 114 families were also screened in a secondary international cohort (details are provided in the Supplemental Material). In total, 189 families containing 209 individuals affected by PCG participated in this study.

LoF gene-burden test. A LoF gene-burden test was performed by considering only the predicted LoF variants (i.e., stop gain, essential splice donor/acceptor, and frameshift insertion/deletion). While 7 of the 189 PCG probands carried heterozygous predicted LoF variants in TEK (p.E150*, p.Y307*, p.T19_R210del, p.K745fs, p.G984*, c. $760+2 \mathrm{~T}>\mathrm{C}$, and c. $3300+2 \mathrm{delT}$ ), only 2 heterozygous LoF TEK variants were present in 60,706 subjects in the ExAC cohort. Since large deletions (e.g., p.T19_R210del in family 2) would not have been called
Figure 8. Model for ANGPT-TEK signal strength and human disease. Schematic model shows the importance of ANGPT-TEK signal strength as a critical determinant of ocular and nonocular vascular phenotypes. In the complete absence of ANGPT-TEK signaling after embryonic day 17.5, SC is not formed and conditional null mice exhibit a severe PCG-like phenotype (31). 50\% reduction of the signal leads to severely hypomorphic SC formation with elevated IOP in mice, and PCG in humans with variable expressivity. In human patients, GoF mutations in TEK result in VMs in nonocular tissues $(34,53,54)$. A frontal view of SC is illustrated in this figure.

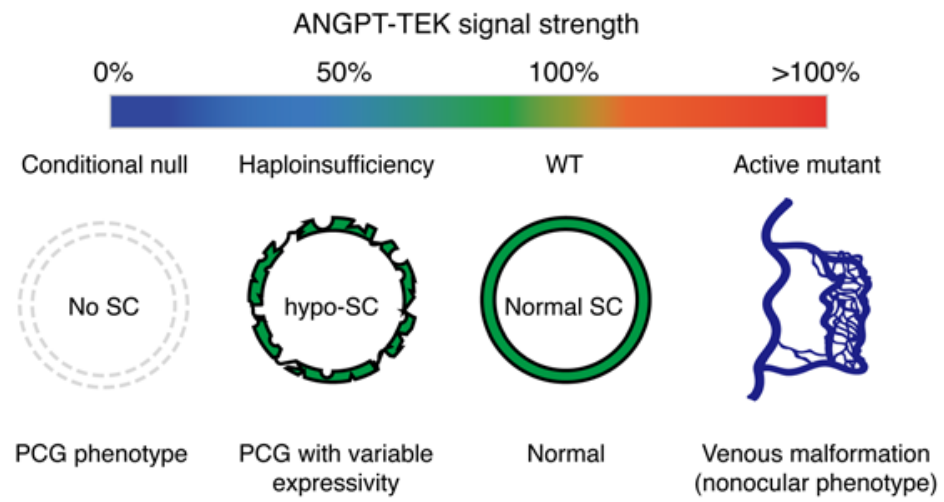


by the VEP/LOFTEE pipeline used by ExAC (https://github.com/ konradjk/loftee), we excluded this variant from our LoF gene-burden analysis. Thereby, we compared 6 of 189 PCG families with 2 of 60,706 individuals in ExAC.

In silico functional studies. PolyPhen-2 $(60,61)$ and SIFT (version 5.2.2) (62) were used to predict any functional effects that TEK missense variants may have on protein function. To analyze the effect of missense variants on protein structure, FoldX (63) analyses were performed using known crystal structures when available. For the two splice donor variants $($ c. $760+2 \mathrm{~T}>\mathrm{C}, \mathrm{c} .3300+2 \mathrm{delT})$, we used HSF version 3.0 (64) and Automatic Analysis of SNP sites (AASsites) (65) to analyze whether the variants can cause changes in splice sites. Detailed protocols are available in the Supplemental Material.

Tissue culture and proteomics. TEK expression constructs were created by cloning full-length TEK cDNA and incorporating a $3^{\prime}$ Flag-tag sequence into the pcDNA3.1 vector. Site-directed mutagenesis and synthesized DNAs were used to generate variant TEK-Flag expression vectors. Total TEK phosphorylation was determined by Western blotting following immunoprecipitation. TEK solubility was analyzed by separating cell lysates into soluble and insoluble fractions. Protein localization studies were performed by transfecting HUVECs with the TEK-Flag vectors and immunostaining using standard protocols. For SILAC analysis, WT TEK-Flag was transfected into light isotope-labeled cells and PCG variants into heavy isotope-labeled cells. The heavy/light ratio of phosphorylated peptides identified by liquid chromatography coupled with tandem mass spectrometry (LC-MS/MS) was calculated by quantification node, and the ratio was normalized by protein abundance. Detailed protocols are available in the Supplemental Material.

Exon trapping splicing assay. Genomic DNA fragments containing TEK exons 5-6 and exon 22 from affected individuals and a control subject were cloned into the exon trapping vector pSPL3. Total RNA from exon trapping construct-transfected Cos-7 cells were reverse-transcribed and PCR-amplified. The resulting RT-PCR products from WT and mutant minigenes were visualized by gel electrophoresis, and their sequence composition was determined by Sanger sequencing. Detailed protocols are provided in the Supplemental Material.

Mice and breeding. Tek WT $\left(\mathrm{Tek}^{+/+}\right)$, hemizygous $\left(\mathrm{Tek}^{+/-}\right)$, and homozygous knockout (Tek cKO) mice were generated by crossing Tek $k^{\text {COIN }}$ mice with ROSA-rtTA;tetO-Cre mice (Supplemental Figure 12). These mouse lines were described previously $(30,31)$. Doxycycline was added to the drinking water of pregnant dams from embryonic day 17.5 to induce Tek deletion. IOP was measured using a TONOLAB rebound tonometer (iCare). TEK expression in lung lysates was analyzed by standard blotting procedures. SC imaging was performed as previously described (31). Mice were maintained on a mixed background, and littermate controls were used for all analyses. Detailed protocols are available in the Supplemental Material.

Statistics. Comparisons of IOP between $\mathrm{Tek}^{+/-}$and WT mice were analyzed by unpaired 2-tailed Student's $t$ test using GraphPad Prism software. The LoF gene-burden test was performed using JMP Pro 11 software, and a two-tailed Fisher's exact test was used for testing the statistical significance. A $P$ value less than 0.05 was considered statistically significant.

Study approval. Subjects and their families were recruited from multiple international centers, each of which had received study approval from their respective institutional review boards: University of Wisconsin-Madison Health Sciences Institutional Review Board; Duke University Health System Institutional Review Board for Clinical Investigations; Children's Hospital of Philadelphia Institutional Review Board; Massachusetts Eye and Ear Infirmary Human Studies Committee; Human Research Ethics Committee of the University of Western Australia; Southern Adelaide Clinical Human Research Ethics Committee, Flinders Medical Centre, Bedford Park, South Australia, Australia; Tasmanian Health and Medical Human Research Ethics Committee, Hobart, Tasmania, Australia; Human Research and Ethics Committee of the Royal Victorian Eye and Ear Hospital; Ethical Review Board of the Medical Faculty of the Friedrich-Alexander University Erlangen-Nürnberg; Ethical Review Board of the Medical Faculty of Niguarda Ca'Granda Hospital; Commission of Ethics for Analysis of Research Projects - Clinical Board of Hospital Clinics and the Faculty of Medicine, University of São Paulo. Written informed consent for study participation was obtained from the subjects or subjects' parents. All animal experiments were approved by the Animal Care Committee of Northwestern University.

\section{Author contributions}

SEQ and TLY conceived, designed, and supervised the study, and wrote the manuscript. TS designed and performed proteomic, cell biology, and comparative biology experiments, analyzed data, and wrote the manuscript. SWT performed and coordinated the human genetic analyses, designed and performed exon trapping experiments, analyzed data, and wrote the manuscript. JJ designed and supervised the proteomics and cell biology study, and wrote the manuscript. JLW supplied patient material, performed human genetic analyses, and wrote the manuscript. FP and JEC supplied patient material and performed human genetic analyses. BRT designed and performed comparative biology experiments, analyzed data, and wrote the manuscript. OMS performed human genetic analyses and wrote the manuscript. VL and SMS performed in silico analyses and wrote a portion of the manuscript. KNW analyzed genetic data and performed experiments. KK and SJ performed experiments and wrote the manuscript. SY, LF, BK, KNTV, and XL performed experiments. LK, DNA, SF, LM, ES, TZ, AWH, KPB, DAM, KFA, JBR, SHL, and SR contributed human subjects, performed genetic analyses, and contributed to writing the manuscript. All authors contributed to the review and approval of the manuscript.

\section{Acknowledgments}

We thank the patient subjects for participating in this study. We also thank John Pater, Joanna Black, Jane Kelly, Sharon Freedman, Ivailo Tournev, and Sylvia Cherninkova for clinical input and Richard Smith for assessment of murine eye phenotypes; Daniel Berner for sequencing assistance; and Andrew Wei and Anna Woo for technical assistance. This study was funded by NIH R01 HL124120 (S.E. Quaggin); NIH R01 EY014685, the Research to Prevent Blindness Inc. Lew R. Wasserman Award, the DukeNational University of Singapore Core Grant, and the University of Wisconsin Centennial Scholars Award (T.L. Young); and NIH Career Development Award K12, 1K23EY020554 (T.L. Yanovitch). This work was also supported by grants from the March of Dimes Foundation (J.L. Wiggs), Howard Hughes Medical Center and EY 11721 (S. John and K. Kizhatil), the Ophthalmic Research 
Institute of Australia, the Channel Seven Children's Research Foundation, Department of Innovation, Industry, Science and Research, and the National Health and Medical Research Council of Australia (NHMRC). T. Souma is supported by fellowship grants from the Japan Society for the Promotion of Science and Mallinckrodt Pharmaceuticals. B.R. Thomson is supported by the Canadian Institute of Health Research. K.P. Burdon, J.E. Craig, and D.N. Azmanov are supported by fellowships from the NHMRC. Imaging was performed at the Northwestern University Center for Advanced Microscopy supported by NCI CCSG P30 CA060553 awarded to the Robert H. Lurie Comprehensive Cancer Center. We also acknowledge support from Vision Core Grant NEI P30EY016665 of the University of Wisconsin-Madison Depart- ment of Ophthalmology and Visual Sciences. Proteomics analyses were supported by the Northwestern University Proteomics Core. We thank Dhaval Nanavati (Northwestern University, Proteomics Core) for technical support and helpful discussions.

Address correspondence to: Terri L. Young, University of Wisconsin-Madison, Department of Ophthalmology and Visual Sciences, 2828 Marshall Ct., Ste. 200, Madison, Wisconsin 53705, USA. Phone: 608.263.9797; E-mail: tyoung6@wisc.edu. Or to: Susan E. Quaggin, Northwestern University, Feinberg Cardiovascular Research Institute and Division of Nephrology/Hypertension, 303 E. Superior St., Suite 10-105, Chicago, Illinois 60611, USA. Phone: 312.503.1531; E-mail: quaggin@northwestern.edu.
1. Weinreb RN, Aung T, Medeiros FA. The pathophysiology and treatment of glaucoma: a review. JAMA. 2014;311(18):1901-1911.

2. Quigley HA. Glaucoma. Lancet. 2011;377(9774):1367-1377.

3. Kwon YH, Fingert JH, Kuehn MH, Alward WL. Primary open-angle glaucoma. $N$ Engl J Med. 2009;360(11):1113-1124.

4. deLuise VP, Anderson DR. Primary infantile glaucoma (congenital glaucoma). Surv Ophthalmol. 1983;28(1):1-19.

5. Sarfarazi M, Stoilov I. Molecular genetics of primary congenital glaucoma. Eye (Lond). 2000;14(pt 3B):422-428.

6. Fan BJ, Wiggs JL. Glaucoma: genes, phenotypes, and new directions for therapy. JClin Invest. 2010;120(9):3064-3072.

7. Gilbert C, Foster A. Childhood blindness in the context of VISION 2020 - the right to sight. Bull World Health Organ. 2001;79(3):227-232.

8. Genĉík A. Epidemiology and genetics of primary congenital glaucoma in Slovakia. Description of a form of primary congenital glaucoma in gypsies with autosomal-recessive inheritance and complete penetrance. Dev Ophthalmol. 1989;16:76-115.

9. Lim SH, et al. CYP1B1, MYOC, and LTBP2 mutations in primary congenital glaucoma patients in the United States. Am JOphthalmol. 2013;155(3):508-517.e5.

10. Allen KF, Gaier ED, Wiggs JL. Genetics of primary inherited disorders of the optic nerve: clinical applications. Cold Spring Harb Perspect Med. 2015;5(7):a017277.

11. Wang R, Wiggs JL. Common and rare genetic risk factors for glaucoma. Cold Spring Harb Perspect Med. 2014;4(12):a017244.

12. Khan AO. Genetics of primary glaucoma. Curr Opin Ophthalmol. 2011;22(5):347-355.

13. Kaur K, et al. Myocilin gene implicated in primary congenital glaucoma. Clin Genet. 2005;67(4):335-340.

14. Cascella R, et al. The genetics and the genomics of primary congenital glaucoma. Biomed Res Int. 2015;2015:321291.

15. Li N, Zhou Y, Du L, Wei M, Chen X. Overview of Cytochrome P450 1B1 gene mutations in patients with primary congenital glaucoma. Exp Eye Res. 2011;93(5):572-579.

16. Stoilov I, Akarsu AN, Sarfarazi M. Identification of three different truncating mutations in cyto- chrome P4501B1 (CYP1B1) as the principal cause of primary congenital glaucoma (Buphthalmos) in families linked to the GLC3A locus on chromosome 2p21. Hum Mol Genet. 1997;6(4):641-647.

17. Libby RT, et al. Modification of ocular defects in mouse developmental glaucoma models by tyrosinase. Science. 2003;299(5612):1578-1581.

18. Bejjani BA, et al. Mutations in CYP1B1, the gene for cytochrome P4501B1, are the predominant cause of primary congenital glaucoma in Saudi Arabia. Am J Hum Genet. 1998;62(2):325-333.

19. Hollands H, Johnson D, Hollands S, Simel DL, Jinapriya $\mathrm{D}$, Sharma S. Do findings on routine examination identify patients at risk for primary open-angle glaucoma? The rational clinical examination systematic review. JAMA. 2013;309(19):2035-2042.

20. Nickells RW, Howell GR, Soto I, John SW. Under pressure: cellular and molecular responses during glaucoma, a common neurodegeneration with axonopathy. Annu Rev Neurosci. 2012;35:153-179.

21. Alm A, Nilsson SF. Uveoscleral outflow - a review. Exp Eye Res. 2009;88(4):760-768.

22. Tamm ER. The trabecular meshwork outflow pathways: structural and functional aspects. Exp Eye Res. 2009;88(4):648-655.

23. Toris CB, Yablonski ME, Wang YL, Camras CB. Aqueous humor dynamics in the aging human eye. Am JOphthalmol. 1999;127(4):407-412.

24. Park DY, et al. Lymphatic regulator PROX1 determines Schlemm's canal integrity and identity. J Clin Invest. 2014;124(9):3960-3974.

25. Kizhatil K, Ryan M, Marchant JK, Henrich S, John SW. Schlemm's canal is a unique vessel with a combination of blood vascular and lymphatic phenotypes that forms by a novel developmental process. PLoS Biol. 2014;12(7):e1001912.

26. Augustin HG, Koh GY, Thurston G, Alitalo K. Control of vascular morphogenesis and homeostasis through the angiopoietin-Tie system. Nat Rev Mol Cell Biol. 2009;10(3):165-177.

27. Barton WA, Dalton AC, Seegar TC, Himanen JP, Nikolov DB. Tie2 and Eph receptor tyrosine kinase activation and signaling. Cold Spring Harb Perspect Biol. 2014;6(3):a009142.

28. Lemmon MA, Schlessinger J. Cell signaling by receptor tyrosine kinases. Cell. 2010;141(7):1117-1134.

29. Thurston G, Daly C. The complex role of angiopoietin-2 in the angiopoietin-tie signaling pathway. Cold Spring Harb Perspect Med. 2012;2(9):a006550.

30. Jeansson M, et al. Angiopoietin-1 is essential in mouse vasculature during development and in response to injury. JClin Invest. 2011;121(6):2278-2289.

31. Thomson BR, et al. A lymphatic defect causes ocular hypertension and glaucoma in mice. J Clin Invest. 2014;124(10):4320-4324.

32. Ali M, et al. Null mutations in LTBP2 cause primary congenital glaucoma. Am J Hum Genet. 2009;84(5):664-671

33. Mears AJ, et al. Mutations of the forkhead/ winged-helix gene, FKHL7, in patients with Axenfeld-Rieger anomaly. Am J Hum Genet. 1998;63(5):1316-1328.

34. Limaye N, et al. Somatic mutations in angiopoietin receptor gene TEK cause solitary and multiple sporadic venous malformations. Nat Genet. 2009;41(1):118-124.

35. Tachibana K, Jones N, Dumont DJ, Puri MC, Bernstein A. Selective role of a distinct tyrosine residue on Tie2 in heart development and early hematopoiesis. Mol Cell Biol. 2005;25(11):4693-4702.

36. Jones $\mathrm{N}$, et al. Identification of Tek/Tie2 binding partners. Binding to a multifunctional docking site mediates cell survival and migration. J Biol Chem. 1999;274(43):30896-30905.

37. Yu X, et al. Structural basis for angiopoietin-1mediated signaling initiation. Proc Natl Acad Sci US A. 2013;110(18):7205-7210.

38. Barton WA, et al. Crystal structures of the Tie2 receptor ectodomain and the angiopoietin-2-Tie2 complex. Nat Struct Mol Biol. 2006;13(6):524-532.

39. Fukuhara S, et al. Differential function of Tie2 at cell-cell contacts and cell-substratum contacts regulated by angiopoietin-1. Nat Cell Biol. 2008;10(5):513-526.

40. Saharinen P, et al. Angiopoietins assemble distinct Tie2 signalling complexes in endothelial cell-cell and cell-matrix contacts. Nat Cell Biol. 2008;10(5):527-537.

41. Fukuhara S, Sako K, Noda K, Nagao K, Miura K, Mochizuki N. Tie2 is tied at the cell-cell contacts and to extracellular matrix by angiopoietin-1. Exp Mol Med.2009;41(3):133-139.

42. Fachinger G, Deutsch U, Risau W. Functional interaction of vascular endothelial-protein- 
tyrosine phosphatase with the angiopoietin receptor Tie-2. Oncogene. 1999;18(43):5948-5953.

43. Economides AN, et al. Conditionals by inversion provide a universal method for the generation of conditional alleles. Proc Natl Acad Sci U S A. 2013;110(34):E3179-E3188.

44. Coleman AL, Miglior S. Risk factors for glaucoma onset and progression. Surv Ophthalmol. 2008;53(suppl 1):S3-S10.

45. Boland MV, et al. Comparative effectiveness of treatments for open-angle glaucoma: a systematic review for the U.S. Preventive Services Task Force. Ann Intern Med. 2013;158(4):271-279.

46. Larsson LI, Rettig ES, Brubaker RF. Aqueous flow in open-angle glaucoma. Arch Ophthalmol. 1995;113(3):283-286.

47. Aspelund A, et al. The Schlemm's canal is a VEGF-C/VEGFR-3-responsive lymphatic-like vessel. J Clin Invest. 2014;124(9):3975-3986.

48. Titheradge H, Togneri F, McMullan D, Brueton L, Lim D, Williams D. Axenfeld-Rieger syndrome: further clinical and array delineation of four unrelated patients with a $4 \mathrm{q} 25$ microdeletion. $A m$ JMed Genet A. 2014;164A(7):1695-1701.

49. Han J, et al. OPA1 mutations and mitochondrial DNA haplotypes in autosomal dominant optic atrophy. Genet Med. 2006;8(4):217-225.

50. Ito YA, Walter MA. Genomics and anterior segment dysgenesis: a review. Clin Experiment Ophthalmol. 2014;42(1):13-24.
51. Lines MA, Kozlowski K, Walter MA. Molecular genetics of Axenfeld-Rieger malformations. Hum Mol Genet. 2002;11(10):1177-1184.

52. Nishimura DY, et al. The forkhead transcription factor gene FKHL7 is responsible for glaucoma phenotypes which map to 6p25. Nat Genet. 1998;19(2):140-147.

53. Vikkula M, et al. Vascular dysmorphogenesis caused by an activating mutation in the receptor tyrosine kinase TIE2. Cell. 1996;87(7):1181-1190.

54 . Wouters V, et al. Hereditary cutaneomucosal venous malformations are caused by TIE 2 mutations with widely variable hyper-phosphorylating effects. Eur J Hum Genet. 2010;18(4):414-420.

55. Macdonald PR, et al. Structure of the extracellular domain of Tie receptor tyrosine kinases and localization of the angiopoietin-binding epitope. J Biol Chem. 2006;281(38):28408-28414.

56. Xu K, et al. Insights into Eph receptor tyrosine kinase activation from crystal structures of the EphA4 ectodomain and its complex with ephrin-A5. Proc Natl Acad Sci U S A. 2013;110(36):14634-14639.

57. Aihara M, Lindsey JD, Weinreb RN. Aqueous humor dynamics in mice. Invest Ophthalmol Vis Sci. 2003;44(12):5168-5173.

58. Lei Y, Overby DR, Boussommier-Calleja A, Stamer WD, Ethier CR. Outflow physiology of the mouse eye: pressure dependence and washout. Invest Ophthalmol Vis Sci. 2011;52(3):1865-1871.
59. Millar JC, Clark AF, Pang IH. Assessment of aqueous humor dynamics in the mouse by a novel method of constant-flow infusion. Invest Ophthalmol Vis Sci. 2011;52(2):685-694.

60. Adzhubei I, Jordan DM, Sunyaev SR. Predicting functional effect of human missense mutations using PolyPhen-2. Curr Protoc Hum Genet. 2013; chapter 7, unit 7.20. doi:10.1002/0471142905.hg0720s76.

61. Adzhubei IA, et al. A method and server for predicting damaging missense mutations. Nat Methods. 2010;7(4):248-249.

62. Sim NL, Kumar P, Hu J, Henikoff S, Schneider G, Ng PC. SIFT web server: predicting effects of amino acid substitutions on proteins. Nucleic Acids Res. 2012;40(Web Server issue):W452-W457.

63. Schymkowitz J, Borg J, Stricher F, Nys R, Rousseau F, Serrano L. The FoldX web server: an online force field. Nucleic Acids Res. 2005;33(Web Server issue):W382-W388.

64. Desmet FO, Hamroun D, Lalande M, Collod-Béroud G, Claustres M, Béroud C. Human Splicing Finder: an online bioinformatics tool to predict splicing signals. Nucleic Acids Res. 2009;37(9):e67.

65. Faber K, Glatting KH, Mueller PJ, Risch A, Hotz-Wagenblatt A. Genome-wide prediction of splice-modifying SNPs in human genes using a new analysis pipeline called AASsites. BMC Bioinformatics. 2011;12(suppl 4):S2. 